\title{
Substitution Effects on the Magnetic Properties of Fe-Containing Chalcogenides with NiAs-Type Structures
}

\author{
N.V. BAranov ${ }^{a, b, *}$, N.V. Selezneva ${ }^{b}$, E.M. Sherokalova ${ }^{b}$, A.F.GubKin $^{a, b}$, \\ A.A. Sherstobitov ${ }^{a, b}$ AND D.A. ShishKin ${ }^{a, b}$ \\ ${ }^{a}$ M.N. Miheev Institute of Metal Physics, Ural Branch of RAS, 620990, Ekaterinburg, Russia \\ ${ }^{b}$ Institute of Natural Sciences and Mathematics, Ural Federal University, 620083, Ekaterinburg, Russia
}

The changes in the magnetization processes caused by Se for S substitutions in the layered chalcogenide compounds $\mathrm{Fe}_{x} \mathrm{TiS}_{2-y} \mathrm{Se}_{y}(x=0.5,0.66)$ with antiferromagnetic (AF) and ferrimagnetic (FI) orderings, respectively, have been studied by using the magnetization and magnetoresistance measurements. Unusually high values of the coercive fields $\left(H_{c}\right.$ up to $56 \mathrm{kOe}$ ) have been observed at low temperatures in $\mathrm{Fe}_{0.5} \mathrm{TiS}_{2-y} \mathrm{Se}_{y}$ with the Se content $y<0.5$, which is ascribed to the presence of an unquenched orbital moment on Fe ions and to the formation of a magnetically heterogeneous state in the vicinity of $H_{c}$. The Se for $\mathrm{S}$ substitution in $\mathrm{Fe}_{0.66} \mathrm{TiS}_{2-y} \mathrm{Se}_{y}$ leads to the transition from the FI to AF state and to non-monotonous change of $H_{c}$ with a maximal value $22 \mathrm{kOe}$ an $y=0.5$. The changes in the magnetic state of $\mathrm{Fe}_{x} \mathrm{TiS}_{2-y} \mathrm{Se}_{y}$ are suggested to be strongly affected by the distribution of $\mathrm{Fe}$ ions and vacancies in cationic layers.

DOI: 10.12693/APhysPolA.133.447

PACS/topics: $75.30 . \mathrm{Kz}, 75.50 . \mathrm{Vv}, 75.60 .-\mathrm{d}$

\section{Introduction}

In iron deficient chalcogenides $\mathrm{Fe}_{1-z} \mathrm{X}(\mathrm{X}=\mathrm{S}, \mathrm{Se})$ with a layered crystal structure of the NiAs-type, the iron layers are sandwiched between completely filled layers of chalcogen. Because of iron deficiency the cationic layers contain vacancies. The $\mathrm{Fe}$ atoms in $\mathrm{Fe}_{1-z} \mathrm{X}$ may be substituted with other $3 \mathrm{~d}$ metal $(\mathrm{M})$ atoms. The magnetic order in chalcogenides $(\mathrm{Fe}, \mathrm{M})_{1-z} \mathrm{X}(z=0.125-0.5)$ is governed by intralayer and interlayer exchange interactions and influenced by distribution of vacancies between cationic layers [1-3]. The magnetic behavior of the $(\mathrm{Fe}, \mathrm{M})_{1-z} \mathrm{X}$ compounds is observed to be dependent on the Fe concentration and on the type and concentration of substituting M' atoms [2,3].

In the $\mathrm{Fe}_{0.5} \mathrm{TiX}_{2}\left(\mathrm{FeTi}_{2} \mathrm{X}_{4}, z=0.25\right)$, one cationic layer is fully occupied by $\mathrm{Ti}$ atoms and the second one is half filled with iron atoms. According to neutron diffraction both the $\mathrm{Fe}_{0.5} \mathrm{TiS}_{2}$ and $\mathrm{Fe}_{0.5} \mathrm{TiSe}_{2}$ compounds exhibit an antiferromagnetic (AF) order below $\sim 140 \mathrm{~K}[4,5]$. The $\mathrm{Fe}_{0.5} \mathrm{TiS}_{2}$ compound was observed to undergo a metamagnetic phase transition from $\mathrm{AF}$ to the ferromagnetic $(\mathrm{F})$ state when the magnetic field is applied below $T_{N}$ [5]. At low temperatures, the fieldinduced $\mathrm{AF}-\mathrm{F}$ transition is found to be irreversible, and the magnetization reversal in the field-induced $\mathrm{F}$ state is accompanied by appreciable hysteresis. According to the neutron diffraction study the $\mathrm{Fe}_{0.5} \mathrm{TiS}_{2-y} \mathrm{Se}_{y}$ compounds with high Se concentrations $(y>0.5)$ exhibit the AF structure with the doubled magnetic unit cell, while the compounds with $y<0.5$ show the AF order with the quadruplicated magnetic unit cell along $a$ and $c$ crystallographic directions of the monoclinic crystal lattice [5].

*corresponding author; e-mail: baranov@imp.uran.ru
The present work aims to study how the Se for S substitution affects the magnetization processes in the compounds of the $\mathrm{Fe}_{x} \mathrm{TiS}_{2-y} \mathrm{Se}_{y}$ type with different Fe concentrations $(x=0.5,0.66)$ and different ground magnetic states.

\section{Experimental}

The $\mathrm{Fe}_{x} \mathrm{TiS}_{2-y} \mathrm{Se}_{y}$ compounds $(x=0.5,0.66)$ were prepared by solid-state reactions inside a sealed quartz tubes in two stages. The starting materials were small pieces of $99.95 \%$ pure iodide titanium, sulfur (99.99\%), selenium (99.99\%) and powder of carbonyl iron (99.98\%). At first, the parent compounds $\mathrm{TiS}_{2-y} \mathrm{Se}_{y}$ were synthesized by heat treatment of a mixture of starting materials at $800^{\circ} \mathrm{C}$ for one week. In the second stage the mixtures of $\mathrm{Fe}$ and $\mathrm{TiS}_{2-y} \mathrm{Se}_{y}$ powders were pressed into cylindrical pellets and annealed at the same conditions. The obtained specimens were milled, compacted into tablets and then homogenized for two weeks at $800^{\circ} \mathrm{C}$, followed by cooling down through removal of the tube from the oven into air. The samples were examined by powder Xray diffraction analysis by using a Bruker D8 Advance diffractometer with $\mathrm{Cu} \mathrm{K} \mathrm{K}_{\alpha}$ radiation. For the refinement of the crystal structure the program FullProf was used. The magnetization measurements were performed by means of MPMS and PPMS systems (Quantum Design, USA). The electrical resistivity were measured from $4 \mathrm{~K}$ up to $300 \mathrm{~K}$ by a four-contact ac method by using a cryo-free DMS-1000 system (Dryogenic Ltd, UK) in magnetic fields up to $10 \mathrm{~T}$.

\section{Results and discussion}

The crystal structure of all the $\mathrm{Fe}_{x} \mathrm{TiS}_{2-y} \mathrm{Se}_{y}$ samples with $x=0.5$ and $x=0.66$ was identified at room temperature as of monoclinic type ( $I 12 / \mathrm{m}$ space group). The Se for $\mathrm{S}$ substitution is observed to result in the expansion 


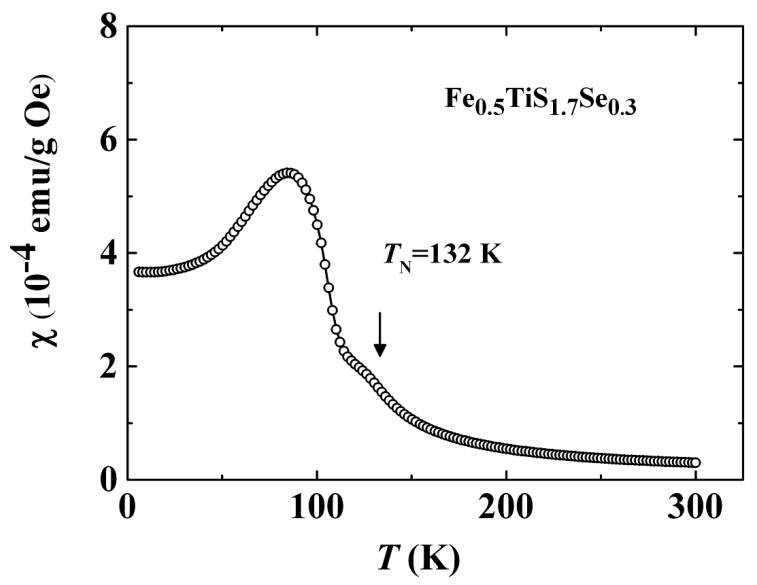

Fig. 1. Temperature dependence of the magnetic susceptibility for $\mathrm{Fe}_{0.5} \mathrm{TiS}_{1.7} \mathrm{Se}_{0.3}$ measured at $H=100$ Oe.

of the crystal lattice because of a larger ionic radius of selenium in comparison with sulfur.

The magnetization measurements performed for the $\mathrm{Fe}_{0.5} \mathrm{TiS}_{1.7} \mathrm{Se}_{0.3}$ compound in the temperature interval from $2 \mathrm{~K}$ up to $300 \mathrm{~K}$ have revealed two anomalies. Bearing in mind the data for the non-substituted $\mathrm{Fe}_{0.5} \mathrm{TiS}_{2}$ compound $[4,5]$ the anomaly observed at $T \approx 132 \mathrm{~K}$ may be associated with the phase transition from the paramagnetic state to the AF order with a non-commensurate magnetic structure which transforms to the commensurate $\mathrm{AF}$ structure with further cooling below $\sim 85 \mathrm{~K}$.

As follows from Fig. 2, application of a magnetic field above $40 \mathrm{kOe}$ leads to the sharp growth of the magnetization. Saturation of the magnetization is not achieved even in the field of $9 \mathrm{~T}$. When the field is varied within $\pm 9 \mathrm{~T}$, a wide hysteresis loop is observed despite the AF ordering. The coercive field, $H_{c}$, reaches a giant value about of $56 \mathrm{kOe}$ at $2 \mathrm{~K}$. In the vicinity of $H_{c}$, the magnetization shows a jump-like behavior at $2 \mathrm{~K}$, while at higher temperatures, a gradual change in the magnetization with a magnetic field is observed.

The magnetoresistance measurements have revealed the irreversibility of the AF-F transition in $\mathrm{Fe}_{0.5} \mathrm{TiS}_{1.7} \mathrm{Se}_{0.3}$ under application of the magnetic field. As follows from Fig. 2b, the AF-F transition is accompanied by a substantial decrease of the electrical resistivity; the magnetoresistance $\Delta \rho / \rho=(\rho(0)-\rho(H)) / \rho(0)$ reaches $-11.5 \%$ at $100 \mathrm{kOe}$, while after switching off the field the magnetoresistance value does not return to its initial value. The inducement of a metastable $\mathrm{F}$ state was also evidenced in the $\mathrm{Fe}_{0.5} \mathrm{TiS}_{2}$ compound by means of neutron diffraction and magnetoresistance measurements $[4,5]$. As follows from Fig. 2, an increase in the field in the opposite direction leads to a decrease of the absolute value of $\Delta \rho / \rho$ in fields of about $50 \mathrm{kOe}$. i.e. in the vicinity of the coercive field. The fact that the resistivity of $\mathrm{Fe}_{0.5} \mathrm{TiS}_{1.7} \mathrm{Se}_{0.3}$ at $H \sim H_{c}$ does not differ substantially from the initial value in the AF state allows us to suggest the formation of a magnetically heterogeneous state. In this inhomogeneous magnetic state, the sample appears to be a mixture of the $\mathrm{F}$ and AF regions, which results in the enhanced values of $H_{c}$ assumingly due to the exchange coupling effect at the interphase boundaries. The compounds $\mathrm{Fe}_{0.5} \mathrm{TiS}_{2-y} \mathrm{Se}_{y}$ with the Se contents $y>0.5$ do not show such a behavior.

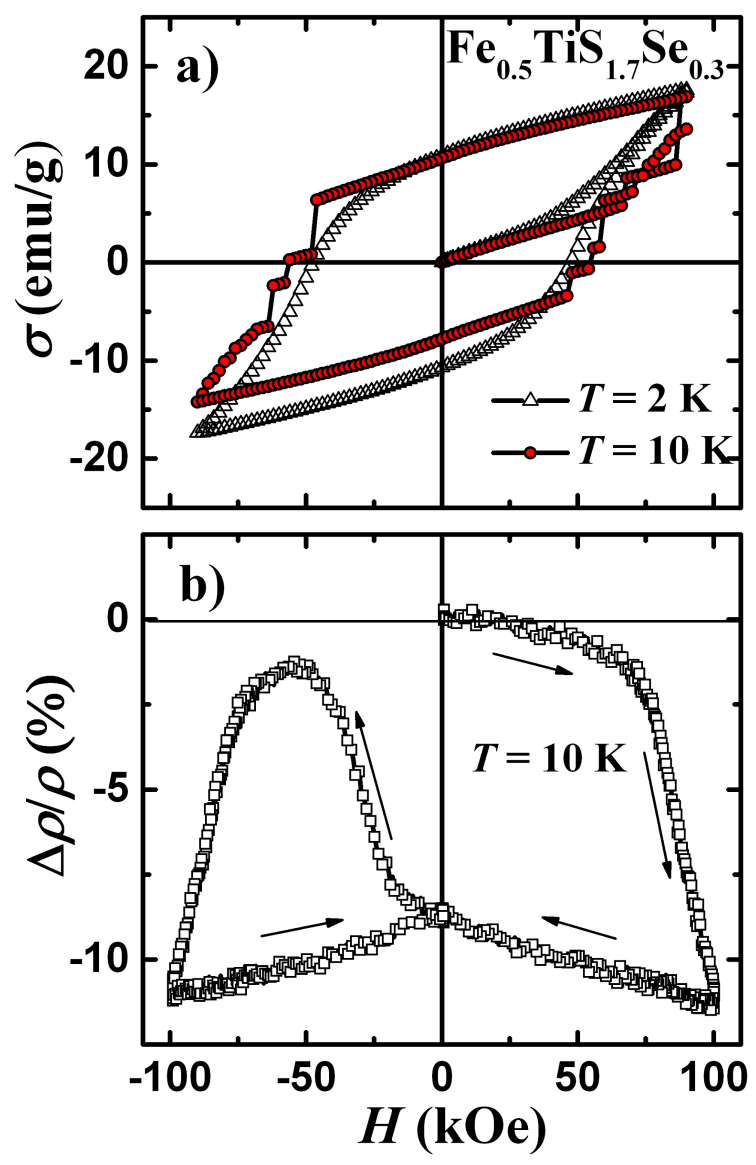

Fig. 2. Field dependences of the magnetization at $2 \mathrm{~K}$ and $10 \mathrm{~K}$ (a) and magnetoresistance at $10 \mathrm{~K}$ (b) for the $\mathrm{Fe}_{0.5} \mathrm{TiS}_{1.7} \mathrm{Se}_{0.3}$ samples.

Unlike $\mathrm{Fe}_{0.5} \mathrm{TiS}_{2-y} \mathrm{Se}_{y}$ compounds exhibiting an $\mathrm{AF}$ ground state in the whole concentration range $(0 \leqslant$ $y \leqslant 2)[5]$, the magnetic state of the sulfide compound $\mathrm{Fe}_{0.66} \mathrm{TiS}_{2}(y=0)$ can be characterized as a ferrimagnetic (FI) one, while the compounds $\mathrm{Fe}_{0.66} \mathrm{TiS}_{2-y} \mathrm{Se}_{y}$ with $y>0.5$ show an $\mathrm{AF}$ behaviour.

The effect of the Se for S substitution in this system can be seen from Fig. 3 which displays the temperature dependences of the magnetic susceptibility, $\chi(T)$, for $\mathrm{Fe}_{0.66} \mathrm{TiS}_{2}$ and $\mathrm{Fe}_{0.66} \mathrm{TiSSe}$. In the case of $\mathrm{Fe}_{0.66} \mathrm{TiS}_{2}$, the sharp change of the magnetic susceptibility at $\sim 160 \mathrm{~K}$ (see Fig. 3a) is associated with the phase transition from the FI order to the paramagnetic state with increasing temperature. Ferrimagnetism in $\mathrm{Fe}_{0.66} \mathrm{TiS}_{2}$ originates in the absence of compensation of the magnetic moments of $\mathrm{Fe}$ atoms located in neighbour cationic layers. The Fe and $\mathrm{Ti}$ ions and vacancies in $\mathrm{Fe}_{0.66} \mathrm{TiS}_{2}$ are suggested to be distributed non-randomly in alternating cationic layers as was observed in $(\mathrm{Fe}, \mathrm{Ti})_{1-z} \mathrm{X}(z=0.125)[2]$. 

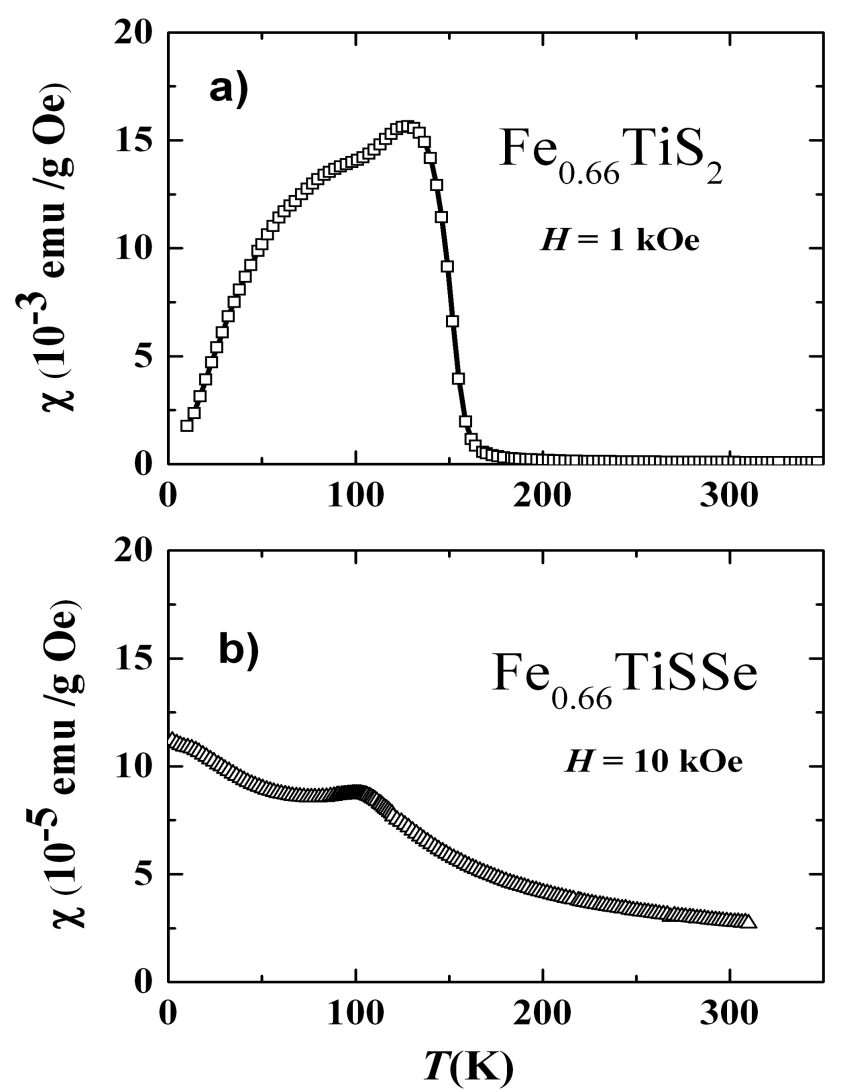

Fig. 3. Temperature dependences of the magnetic susceptibility for $\mathrm{Fe}_{0.66} \mathrm{TiS}_{2}$ (a) and $\mathrm{Fe}_{0.66} \mathrm{TiSSe}(\mathrm{b})$.

The anomaly at $\sim 100 \mathrm{~K}$ on the $\chi(T)$ dependence for $\mathrm{Fe}_{0.66}$ TiSSe (Fig. 3b) indicates the phase transition from AF to the paramagnetic state with increasing temperature. The changes in magnetic behavior in the $\mathrm{Fe}_{0.66} \mathrm{TiS}_{2-y} \mathrm{Se}_{y}$ system from FI to AF with the Se for $\mathrm{S}$ substitution apparently results from an increased disorder in the cationic subsystem, i.e. from a more statistical distribution of $\mathrm{Fe}$ ions and vacancies between layers. Bearing in mind an AF coupling of the Fe magnetic moments belonging to the neighbor layers the random distribution of $\mathrm{Fe}$ ions and vacancies in layers implies a zero net magnetization.

Figure 4. shows how the Se for $\mathrm{S}$ substitution in $\mathrm{Fe}_{0.66} \mathrm{TiS}_{2-y} \mathrm{Se}_{y}$ affects the magnetization versus field behavior at $2 \mathrm{~K}$. The replacement of $\mathrm{S}$ ions by Se leads to decrease of the resultant magnetization and substantially enhances the coercive field from $H_{c}=8 \mathrm{kOe}$ at $y=0$ up to $H_{c}=22 \mathrm{kOe}$ at $y=0.5$.

\section{Conclusions}

The layered chalcogenide compounds $\mathrm{Fe}_{x} \mathrm{Ti}(\mathrm{S}, \mathrm{Se})_{2}$ with the different Fe concentrations $(x=0.5,0.66)$ have been synthesized and studied. At low temperatures, the AF ordered compounds $\mathrm{Fe}_{0.5} \mathrm{TiS}_{2-y} \mathrm{Se}_{y}(y<0.5)$ exhibit the field-induced phase transitions to the metastable $\mathrm{F}$ state with a giant coercivity $\left(H_{c}\right.$ up to $\left.56 \mathrm{kOe}\right)$. Such a magnetic hardness together with the presence of unquenched orbital moment on Fe ions is suggested to result from heterogeneous magnetic state realizing in the

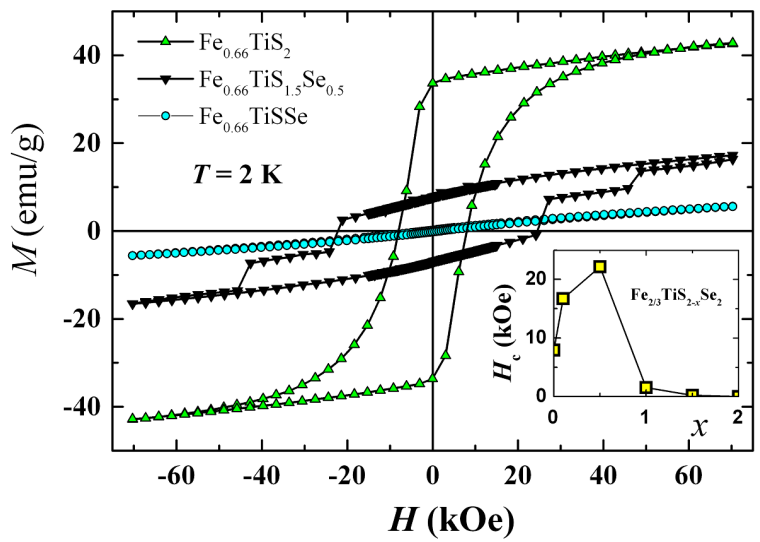

Fig. 4. Field dependences of the magnetization measured at $2 \mathrm{~K}$ on the $\mathrm{Fe}_{0.66} \mathrm{TiS}_{2-y} \mathrm{Se}_{y}$ compounds with various selenium concentrations.

vicinity of $H_{c}$. As derived from the magnetoresistance measurements, such a magnetic state seemingly consist of both $\mathrm{AF}$ and $\mathrm{F}$ regions. The Se for $\mathrm{S}$ substitution in $\mathrm{Fe}_{0.66} \mathrm{TiS}_{2-y} \mathrm{Se}_{y}$ leads to the transition from the FI to AF state, which is ascribed to the increased disorder in cationic layers. The growth of the Se content in $\mathrm{Fe}_{0.66} \mathrm{TiS}_{2-y} \mathrm{Se}_{y}$ is accompanied by the reduction of the resultant magnetization and by a non-monotonous change of the coercive field with a maximal value of about $22 \mathrm{kOe}$ at low temperatures. Unusually high magnetic hardness observed in $\mathrm{Fe}_{x} \mathrm{Ti}(\mathrm{S}, \mathrm{Se})_{2}$ is a very rare case for the materials the magnetism of which is associated with iron since the iron materials usually exhibit soft magnetic properties.

\section{Acknowledgments}

This work was supported by the RFBR (project No 16-02-00480), by the program of UB of RAS (project No 18-10-2-5) and by the Ministry of Education and Science of Russia (project No 3.2916.2017/4.6).

\section{References}

[1] H. Wang, I. Salveson, Phase transition 78, 547 (2005).

[2] N.V. Baranov, P.N.G. Ibrahim, N.V. Selezneva, A.F. Gubkin, A.S. Volegov, D.A. Shishkin, L. Keller, D. Sheptyakov, E.A. Sherstobitova, J. Phys.: Cond. Matt. 27, 286003 (2015).

[3] M. Inoue, H. Hughes, A. Yoffe, Adv. Phys. 38, 565 (1989).

[4] N.V. Baranov, E.M. Sherokalova, N.V. Selezneva, A.V. Proshkin, A.F. Gubkin, L. Keller, A.S. Volegov, E. P. Proskurina, J. Phys.: Cond. Matt. 25, 066004 (2013).

[5] A.F. Gubkin, E.M. Sherokalova, L. Keller, N.V. Selezneva, A.V. Proshkin, E.P. Proskurina, N.V. Baranov, J. Alloys Comp. 616, 148 (2014). 